\title{
Most download AXA papers in 2012
}

In early March I received an email from ICDD, informing me of the results of a statistical analysis of the most download AXA papers. The main contents of the ICDD email are as follows:

“... The International Centre for Diffraction Data has posted Advances in X-ray Analysis, the proceedings for the Denver X-Ray Conference, on-line for several years as a free download.

We recently completed a statistical download analysis from our website and Dr. Lin's Volume 41 paper, of which you were a co-author, Peak Identification of Conventional X-ray Diffraction Patterns for MBE FePt Thin Films on MgO Single-crystal Substrates, was downloaded 1732 times in just the past year OUTSTANDING! You have the 11th highest download frequency from among the more than 1000 publications on our website. Your paper is now listed on "Most Frequent Downloads" on our site.

This type of download activity is not tracked by conventional citation services so we thought you would like to know the practical consequence of writing this publication. Please share this information with your co-authors ...."

I was amazed to find out from the ICDD email that one of the papers in Volume 41 published in 1997, of which I was a co-author, was downloaded amazingly more than 1700 times in just the past year. I am also happy to learn that at least 10 other AXA papers were downloaded more than my paper. The results of this statistical analysis reveal the popularity and the quality of the AXA papers. Congratulation to the Denver X-ray Conference and the Conference sponsor, ICDD, for doing an excellent job on publishing and promoting the Advances in X-ray Analysis.

A list of the 30 most frequent download AXA papers in 2012 (updated by ICDD in February 2013) are as follows:

\section{XRD - Most Frequent Downloads}

X-ray Diffraction Analysis in the Forensic Science: The Last Resort in Many Criminal Cases

Fundamentals of Two-Dimensional X-ray Diffraction (XRD2)

Advances in Quantitative XRD Analysis for Clinker, Cements, and Cementitious Additions - new to list

Applications of X-ray Diffraction in Conservation Science and Archaeometry

Powder X-ray Diffraction Detection of Crystalline Phases in Amorphous Pharmaceuticals - new to list

Peak Identification of Conventional X-ray Diffraction Patterns for MBE FePt Thin Films on MgO Single-crystal Substrates

Apatite Structures - new to list

Microstructure of Clay-Polymer Composites

Strain Broadening Caused by Dislocations - new to list
Grazing Incidence in-plane X-ray Diffraction in the Laboratory - new to list

Quantitative Analysis of Calcium Oxide Desiccant Conversion to Calcium Hydroxide Using X-ray Diffraction - new to list

X-ray Diffractometry Studies and Lattice Parameter Calculation on $\mathrm{KNO}_{3}-\mathrm{NH}_{4} \mathrm{NO}_{3}$ Solid Solutions - new to list

A Historical Review of Retained Austenite and its Measurement by X-ray Diffraction - new to list

XRF - Most Frequent Downloads

XRF Analysis - Theory, Experiment, and Regression Fundamental Parameter Methods in XRF Spectroscopy

Problems by Using Pressed Powder Pellets for XRF Analysis of Ferrosilicon Alloys

Approaching a Universal Sample Preparation Method for XRF Analysis of Powder Materials

Elemental Imaging for Pharmaceutical Tablet Formulation Analysis by Micro X-ray Fluorescence - new to list

Applications and Perspectives of a New Innovative XRF-XRD Spectrometer in Industrial Process Control

Spectral Interference in X-ray Fluorescence Analysis of Common Materials - new to list

ED-XRF Spectrum Evaluation and Quantitative Analysis Using Multivariate and Nonlinear Techniques

\section{X-ray Methods - Most Frequent Downloads}

A Close Look at Electrolytic Manganese Dioxide (EMD) and the $\gamma-\mathrm{MnO}_{2} \& \varepsilon-\mathrm{MnO}_{2}$ Phases Using Rietveld Modelingnew to list

X-ray Backscatter Imaging: Photography through Barriers - new to list

Carbon-Nanotube Field Emission X-ray Tube for Space Exploration XRD/XRF Instrument

$\mathrm{X}$ rays in art and archaeology: an overview - new to list

Thin Film Density Determination by Multiple Radiation Energy Dispersive X-ray Reflectivity

Profile Fitting and Diffraction Line Broadening Analysis X-ray Optics - new to list

A Direct Algorithm for Solving Ill-Conditioned Linear Algebraic Systems - new to list

Mineral Classification in the Mineral Powder Diffraction File

The above 30 most popular papers cover a wide variety of important and useful applications of X-ray analysis. Please visit the 30 most frequently download AXA papers posted on line on the "Advance in X-ray Analysis" page of the ICDD website for free download. I also would like to point out that more than 1000 AXA papers published in Volume 40 (1996) to 53 (2011) of AXA have also posted on line on the "Advance in X-ray Analysis" page of the ICDD website for free download. I hope that you will find the AXA papers interesting and useful.

Ting C. Huang

Editor-in-Chief 